\title{
Recombinant Poliovirus circulation among healthy children immunized with oral polio vaccine in Abidjan
}

\author{
Akoua-Koffi GC ${ }^{1 *}$, Gouandjika $\mathrm{I}^{2}$, Tieoulou $\mathrm{L}^{1}$, Faye-Kette $\mathrm{H}^{1}$, Morvan $\mathrm{J}^{2}$, Dosso $\mathrm{M}^{1}$ and \\ Ehouman $A^{1}$
}

\author{
'Laboratoire des Entérovirus, Institut Pasteur de Côte d'Ivoire 01 BP 490 Abidjan, Côte d'Ivoire. \\ ${ }^{2}$ Laboratoire Régional de Référence OMS pour la poliomyélite, Institut Pasteur de Bangui BP 936, Bangui (RCA)
}

Accepted 1 April, 2004

In order to assess the level of polio virus with natural recombinant genome and wild polio virus circulating in the environment of healthy children aged 0 to 5 years in Abidjan, 130 polio viruses made up of 26 polio type 1, 55 type 2 and 49 type 3 were identified by neutralisation test with monoclonal antibodies and restriction fragment length polymorphism (RFLP) targeting the VP1 and 3D1 gene. Four wild non Sabin-like (NSL) strains (3.1\%): one type 2 and three type 3 were identified in non vaccinated children. One hundred and six $(81.5 \%)$ isolates were Sabin-like, $20(15.4 \%)$ were recombinant with the following polio virus profiles: 2 Sabin-like type 1/type 2, 3 Sabin-like type 3/type 1, 11 Sabin-like type $3 /$ type 2 and one polio virus type 3 NSL/Sabin-like type 3 . Intertypic vaccine/vaccine or vaccine/wild strain recombinant polio virus circulating among healthy children rate was high and suggested the need for a molecular surveillance of vaccine strains. Oral Polio Vaccine (OPV) strains are well-known to revert to pathogenicity in vaccines. Therefore, the long term excretion of pathogenic OPV derived strains by some vaccinees needs to be considered quite seriously. It therefore suggested that all polio virus isolated from acute flaccid paralysis (AFP) be analyzed by restriction fragment length polymorphism and sequencing of the viral genome.

Keys words: polio virus, recombinant virus, healthy children, Cote d'Ivoire.

\section{INTRODUCTION}

Côte d'Ivoire has subscribed to the eradication paralytic poliomyelitis program initiated by World Health Assembly (Resolution WHA 41.28). Oral Polio Vaccine (OPV) was successfully used to control paralytic poliomyelitis in temperate climates and some subtropical region of the world, especially the Americas (EPI/PAHO, 1993; EPI, 1994).

Vaccination campaigns, routine immunization and active surveillance programs are needed before the ultimate goal of eradication is achieved. However these vaccine strains raise some problems which can compromise the eradication of poliomyelitis. Mutations and recombination occur frequently with vaccine strains, especially with the less stable types 2 and 3 polio virus, upon replication in the human intestine (Pallansch and Roos, 2001). These strains can also in rare cases revert

*Corresponding author. E-mail: polioci@globeaccess.net or akouamc@yahoo.fr. to neurovirulence. Some cases of acute flaccid paralysis, vaccine associated paralytic poliomyelitis (VAPP) have been described with vaccine strains as reported by Furione et al. (1993) and Georgescu et al. (1995). There is little or no information on the survival rate of the OPV viruses in circulation in Cote d'Ivoire after vaccination. It is therefore important to evaluate the rate of recontamination of the population after mass vaccination campaigns, and assess the risk associated with mutation or recombination of the vaccine strains as observed in earlier findings by Fine and Carneiro (1999).

Before setting up the acute flaccid paralysis (AFP) active surveillance in 1997, studies were held to determine the level of wild polio virus circulating among children, in rural or urban areas during which period many polio viruses were isolated from vaccinated and non vaccinated children in Cote d'Ivoire.

This study is therefore aimed at reporting the level of recombination and wild polio virus circulating among healthy children aged 0 to 5 years in Abidjan in 1996. 
Table 1. Vaccinal status of Poliovirus carriers according to the period of stool collection.

\begin{tabular}{|c|c|c|c|c|c|c|c|c|}
\hline & \multicolumn{2}{|c|}{0 OPV dose } & \multicolumn{2}{|c|}{1 OPV dose } & \multicolumn{2}{|c|}{2 OPV doses } & \multicolumn{2}{|c|}{3 OPV doses } \\
\hline \multicolumn{9}{|c|}{ Vaccination $(n=121)$} \\
\hline Newborns & \multicolumn{2}{|c|}{0} & \multicolumn{2}{|c|}{$60(49.6 \%)$} & \multicolumn{2}{|c|}{$15(12.4 \%)$} & \multicolumn{2}{|c|}{$18(14.9 \%)$} \\
\hline Contacts & \multicolumn{2}{|c|}{$20(16.5 \%)$} & \multicolumn{2}{|c|}{$8(6.6 \%)$} & \multicolumn{2}{|c|}{0} & \multicolumn{2}{|c|}{0} \\
\hline & \multicolumn{2}{|c|}{ PO } & \multicolumn{2}{|c|}{ P1 } & \multicolumn{2}{|c|}{ P2 } & \multicolumn{2}{|c|}{ P3 } \\
\hline Virus $(n=130)$ & Polio & NPE & Polio & NPE & Polio & NPE & Polio & NPE \\
\hline Newborns & 0 & 0 & 66 & 0 & 15 & 9 & 21 & 11 \\
\hline Contacts & - & - & 17 & 12 & 11 & 11 & - & - \\
\hline
\end{tabular}

OPV = Oral Poliovirus Vaccine

$\mathrm{PO}=$ samples collected before OPV

$\mathrm{P} 1$ = samples collected after 1 st dose of OPV

$\mathrm{P} 2$ = samples collected after 2nd dose of OPV

P3 = samples collected after 3rd dose of OPV

$\mathrm{NPE}=$ non-polio enterovirus

\section{MATERIAL AND METHODS}

\section{Population and stool collection}

The study was udertaken in a suburban environment in Abidjan. One hundred and fourty-five newborns were recruited on the basis of the following criteria: delivery in a maternity center, and possibility to monitor the mother and child, and others in the family. Two-hundred and fifty-two contacts made up of brothers, sisters and children living in the commune yard in a cohorte of 3 to 5 per family were enrolled.

Between March and August 1996, samples of freshly produced stools were collected from each child at different periods. For the newborns, stools were collected before OPV and 8 days after each OPV dose according to the immunization timetable. For the contacts, stools were collected during the second and third collecting periods with the newborns. The samples were accompanied by information forms and were forwarded to the Enterovirus Laboratory in Institute Pasteur Cote d'Ivoire (IPCI), in iceboxes, at $0^{\circ}$ and $+4^{\circ} \mathrm{C}$. Samples were stored at $-20^{\circ} \mathrm{C}$ until ready for use.

\section{Isolation and identification}

Stool samples were treated for virus isolation and identication according to the protocol recommended by World Health Organisation (WHO 1990). Briefly described, stools were extracted with chloroform and inoculated into Hep-2 and RD cells and typed by sero-neutralization. The isolation and identification of the isolates were carried out at IPCI Enterovirus Laboratory.

Intratypic differentiation (ITD) was done by monoclonal antibodies (Mab) and by reverse transcriptase polymerase chain reaction (RT-PCR) followed by restriction fragment length polymorphism (RFLP) according to the modified version of Balanant et al. (1991) at the Reference Regional Polio Laboratory, Pasteur institute in Bangui. The PCR targeted two regions:

1. Capsid's VP1 polypeptide gene, a sequence of 472 to 480 nucleotides according to the type of polio virus, using the "downstream" primer UC1 (5'-GAATTCCATGTCCAAATCTAGA-
3') and the "upstream" primer UG1 (5'-GAATTCCATGTCCAAAT CTAGA-3').

2. Polymerase 3D1 region gene, a sequence of 431 nucleotids (6086-6376) amplified with the "downstream" primer UC12 (5'GAATTCCATGTCCAAATCTAGA-3') and "upstream" primer UG7 (5'TTTGAAGGGGTGAAGGAACCAGC-3) with a view to highlighting the genomic recombinations.

Strain-specific restriction profiles were generated using three restriction enzymes, HaellI, Hpall and Ddel for RFLP-1, and HaellI, Rsal and Xbal for RFLP-3D1 as reproted by Guillot et al. (2000), with $1.5 \%$ agarose for the electrophoresis. The reagents used, polyclonal and monoclonal antibodies, primers and restriction enzymes for the RFLP test were provided by the WHO Reference and Research Center for Poliomyelitis of the Paris Institute Pasteur and WHO Regional Reference Polio Laboratory in Bangui.

\section{Statistical analysis}

The EPI/INFO software was used for the statistical study of the results obtained. The Pearson's chi square tests with corrections by YATES were used to compare the populations that were surveyed (0.05 threshold). The exact Fisher test was used for lower numbers.

\section{RESULTS}

\section{Identification}

A total of 918 samples were collected out of which 173 $(18.8 \%)$ enterovirus strains were isolated. These consisted of 130 polio virus $(75.1 \%)$ and $43(24.9 \%)$ non polio-enterovirus (NPEV) from 121 children. Of the 130 polio viruses, 26 (20\%) were type 1, $55(43.3 \%)$ type 2 and $49(37.7 \%)$ were type 3 . Approxymatly $78.5 \%$ of the polio virus were isolated from the newborns and $21.5 \%$ from the contacts $(p<0.01)($ Table I). 


\section{Intratypic differenciation}

Intratypic differentiation of the 130 polio viruses were determined using the monoclonal antibodies technique. Five $(3.8 \%)$ wild or non-Sabin-like (NSL) strains (one type 1 and 4 type 3 ) were isolated from the contacts, three of which have not received any previous OPV dose, while $125(96.2 \%)$ isolates were identified as Sabin-like (SL) strains.

\section{Characterization by RT-PCR/RFLP}

All the 130 polio virus isolates were analyzed by the RTRFLP method. Only 4 of the $5 \mathrm{NSL}$ were identified as NSL by the RT-PCR-RFLP, and were made up of one type 1 and Poliovirus type 2 and 3 Poliovirus type 3 (not shown). The RT-PCR-RFLP identified 106 as SL strains. Nineteen SL and one NSL virus showed recombinant profiles with the RFLP-3D1 region. Sabin-like type 3 strains carried more recombinations $(13 / 20,65 \%)$, than type $2(3 / 20)$ and type $1(2 / 20)$. One NSL type 3, previously identified by the monoclonal antibodies technique turned out to be a recombinant strain wild Poliovirus 3/Sabin-like 3 . The distribution of the recombinants is shown in Table 2. The results of both the ITD by monoclonal antibodies and RFLP test showed that $81.5 \%$ were vaccine strains, $15.4 \%$ recombinant strains while $3.1 \%$ were wild strains.

Table 2. Results of the RFLP profiles analysis.

\begin{tabular}{|l|l|l|}
\hline $\begin{array}{c}\text { Sabin-like (n } \\
\mathbf{= 1 0 6 )}\end{array}$ & $\begin{array}{c}\text { Non Sabin-like } \\
(\mathbf{n}=\mathbf{4})\end{array}$ & \multicolumn{1}{|c|}{$\begin{array}{c}\text { Recombinants } \\
(\mathbf{n}=\mathbf{2 0})\end{array}$} \\
\hline 24 Polio 1 & & $2 \mathrm{~S} 1 / \mathrm{S} 2$ \\
51 Polio 2 & 1 Polio 2 & $3 \mathrm{~S} 2 / \mathrm{S} 1$ \\
31 Polio 3 & 3 Polio 3 & $\begin{array}{l}3 \mathrm{~S} 3 / \mathrm{S} 1 \\
11 \mathrm{~S} 3 / \mathrm{S} 2 \\
1 \text { Polio 3 NSL/S3 }\end{array}$ \\
& &
\end{tabular}

NSL = non Sabin-like

S1 = Poliovirus type 1 Sabin-like

S2 = Poliovirus type 2 Sabin-like

S3 = Poliovirus type 3 Sabin-like

\section{Comparison between ITD by monoclonal antibodies and RT-PCR/RFLP}

One hundred and twenty-five polio virus isolates were identified by monoclonal antibodies as SL type 1 (26 strains), type 2 (54 strains) and type 3 (46 strains) and 5 as NSL. RFLP identified 24 as SL type 1, 51 SL type 2 and $31 \mathrm{SL}$ type 3 . In the same manner the RFLP identified 4 NSL and 20 recombinant strains profiles. The RFLP confirmed the serotype and gave complementary information by pointing out the recombinant strains.

\section{DISCUSSION}

In this study we have attempted to determine the rate of recombinant and wild polio virus circulating among vaccinated and non-vaccinated children in Abidjan. Children carrying poliovirus were newborns aged 2 months to 4 months, a period of full vaccinal activity, hence the high number of excreted vaccinal strains as compared to 1 to 6 years among the contacts. As shown in Table I, on the basis of the vaccination cards available, most of the contacts found positive had not received OPV while only 8 of them have received between the ages of 6 months and 4 years. All the newborns recruted received OPV. Three NSL strains were isolated from non immunized children.

In this study, we have found a carrier rate of $14.2 \%$ among children in Abidjan. This is significantly different from the rate obtained by Gershy-Damet et al. (1986) in Abidjan when he found only $8 \%$ among children aged 6 to 8 years old $(p<0,001)$. This difference could be explained by the fact that in our study only contacts of vaccinees were enrolled. The majority of isolates were vaccine serotype 2 (49\%). This is in aggrement with the finding of Krugman et al. (1960). The administration of trivalent vaccine is indeed followed mainly by excretion containing serotype 2 as reported by Fine and Carneiro (1999). However, the persistence of excretion after the $3^{\text {rd }}$ dose raises questions as to the future of vaccinal strains.

Among the strains analyzed, $81.5 \%$ were of vaccinal origin, $3.1 \%$ were wild Poliovirus strains and $15.4 \%$ were recombinants. Natural modifications in the genome is a normal evolution for most RNA genomic viruses out of which polio virus is one. This property is more pronounced especially among the vaccine strains. The genome of the vaccine strains can carry out mutation and recombination processes, and those recombinant strains can be responsible for acute flaccid paralysis. Although the biological significance of frequent occurence of recombinants among field isolates of vaccine-related strains was discussed by Lipskaya et al. (1991), many authors (Beletskaia et al., 2002; Furione et al., 1993; Friedrich et al., 1996) have reported recombinant strains isolated from vaccine-associated paralytic poliomyelitis cases, some with a high proportion of $50 \%$ (Guillot et al., 2000).

The recombinant vaccine strains should be investigated on molecular aspects, sequencing the VP1 capsid and RNA polymerase regions. Intertypic vaccine/vaccine or vaccine/non recombinant Poliovirus rate $15.4 \%$ obtained in this study seemed high among these apparently healthy children, particulary in those without a strong immunity during the immunization period or even after the three OPV doses. Serotype 3 is considered as the least immunogenic among the vaccinal strains as shown by many serological studies (Akoua et al., 1995; Sabin, 1984). It would have been advisable to study the immune status of all the children who excreted Poliovirus after 
three doses of OPV. An incomplete or absence of immune response after the three OPV doses might explain an unapparent infection by a vaccinal or wild strain.

Recombinant polio virus strains excretion and circulation among non or insufficiently immunized populations might constitute a risk for the Polio eradication program because of potential epidemics of poliomyelitis that may occur. It therefore seems important to get high immunization coverages in the first year of live (0-11 months), specially in the developping countries because of the contact with other eterovirus infection in early life. In $75 \%$ cases of the recombinant strains, Sabin-like type 3 was implicated, $20 \%$ with type 2 and $10 \%$ with type 1 . It is known that type 3 vaccine strains contains less attenuated mutations compared with the type 1 and type 2 strains of OPV. Moreover, OPV strains are well-known to revert to pathogenicity in vaccinees, therefore the long term excretion of pathogenic OPV derived strains by vaccinees needs to be considered quite seriously.

Despite immunization by OPV, wild polio virus were circulating among children but at a lower level. This fact justifies the vast immunization campaigns, in form of national immunization days (NIDs) in Cote d'Ivoire since 1997. Wild polio virus circulation among healthy children immunized with OPV is also reported by Andrianarivelo et al. (1999) in Antananarivo, Madagascar. This situation is common if the immunization coverage is low, and the titres of specific antibodies against polio virus are insufficient to protect against new Poliovirus infections. Two of the 5 children carrying a wild virus strains had received one dose recommended at 1 and 2 months old. Their immunity did not prevent intestinal carriage of wild polio virus strains.

From the virological point of view, the RT-PCR/RFLP technique allowed a better intratypic discrimination because it revealed the structural modifications of the viral genomes which were not identified by the monoclonal antibodies as earlier described by Crainic and Kew (1993). However the use of RFLP tests has made it possible to discover recombinations. Nineteen recombinant strains and the NSL/SL strain were identified as SL and NSL using monoclonal antibodies. The study relating to these strains will be completed by the analysis of the nucleotide sequences of the amplified VP1 region to determine the nature of the changes observed in RFLP.

Intertypic vaccine/vaccine or vaccine/wild strain recombinant Poliovirus circulation among healthy children is frequent and suggest a molecular surveillance. Routine and mass immunization campaigns provide a lot of vaccine strains in circulation. These campaigns are usefull to eradicate wild Poliovirus but there is a risk of spreading of the genetically modified OPV strains which could be associated with illnesses such as paralytic poliomyelitis. It is therefore suggested that all polio virus isolated from acute flaccid paralysis cases should be analyzed by restriction fragment length polymorphism and sequencing.

\section{AKNOWLEGEMENTS}

The work presented in this article was supported by grants from the Délégation Générale du Réseau Internatinal des Instituts Pasteur et Instituts Associés" of the Institut Pasteur, Paris. We thank Dr. Radu Crainic for the reagents and Ms. Sophie Guillot and Dr. Francis Delpeyroux for usefull technical advices. We also thank Prof. F. D. Adu for reading the manuscript.

\section{REFERENCES}

Akoua-Koffi G, Thonnon J, Kouassi-Renaud M, Dosso M, Ehouman A (1995). Séroprévalence antipoliomyélitique post-vaccinale en milieu urbain à Abidjan. Bull. Soc. Pathol. Exot. 88: 117-120.

Andrianarivelo MR, Rabarrijaona L, Boisier P, Chezzi C, Zeller HG (1999). Wild Poliovirus circulation among healthly children immunized with oral polio vaccine in Antananarivo, Madagascar. Trop. Med. Int. Health 4(1): 50-57.

Balanant J, Guillot S, Candrea A, Delpeyroux F, Crainic R (1991). The natural genomic variability of Poliovirus analyzed by Restriction Fragment Length Polymorphism assay. Virology 184: 645 - 654 .

Beletskaia TS, Samoilovich EO, Korotkova EA, Scheslenok EP, Fel'dman EV (2002). Mutation and recombination variability of vaccine poliovirus isolated in Belorus (1960-1999). Mol. Gen. Mikrobiol. Virusol. 1: 24-31

Crainic R, Kew O (1993). Evolution and polymorphism of poliovirus genomes. Biologicals 21: $379-384$.

EPI (1994). Certification of poliomyelitis eradication-the Americas. WER 69: 293-295.

EPI/PAHO (1993). Strategies for the certification of the eradication of wild poliovirus transmission in the Americas. Bulletin of PAHO 27: 287-296.

Fine PEM, Carneiro IAM (1999). Transmissibility and persistence of oral polio vaccine viruses: implications for the global poliomyelitis eradication initiative. Am. J. Epidemiol. 150: 1001-1021.

Furione M, Guillot S, Otela D, Balanant J, Candrea A, Crainic R (1993). Polioviruses with natural recombinant genomes isolated from vaccine-associated paralytic poliomyelitis. Virology 196 (1): 199-208.

Friedrich F, Da-Silva EF, Schatzmayr HG (1996). Type 2 poliovirus recombinants isolated from vaccine-associated cases and from healthly contacts in Brazil. Acta Virol. 40 (1): 27-33.

Georggescu MM, Delperoux F, Craainic R (1995). Tripartite genome organization of a natural type 2 vaccine/non vaccine recombinant poliovirus. J. Gen. Virol. 76 (Part 9): 2343-2348.

Gershy-Damet G.M., Sangaré G.A., Penali K.L., Affian K. Abloh A (1986). Prévalence du virus de la poliomyélite dans les communes d'Abidjan. Méd. Afr. Noire 33 (12): $873-875$.

Guillot S, Caro V, Cuevo N et al (2000). Natural genetic exchanges between vaccine and wild poliovirus strains in humans. J. Virol. 74 (18), 8434-8443.

Krugman S, Warren J, Eiger MS, Bernam PH, Michaels RH, Sabin AB (1960). Immunization of newborns infants with live attenuated poliovirus vaccines. Second International Conference on live Poliovirus vaccine, Washington.

Lipskaya GY, Muzychenko AR, Kutitova OK et al (1991). Frequent isolation of intertypic poliovirus recombinants with serotype 2 specificity from vaccine-associated polio cases. J. Med. Virol. 35 (4): 290-296.

Pallansch MA, Roos RP (2001). Enteroviruses: Poliovirus, Coxsackievirus, Echovirus and never Enteroviruses. In Virology (1); Fields, $4^{\text {th }}$ Ed, Lippincott, Williams and Wilkins, pp. 723-775. 
Sabin AB (1984). Stategies for elimination of poliomyelitis in different parts of the world with use of oral poliovirus vaccine. Rev. Infect. Dis.; 27: 287-296.

WHO (1996). Progress towards poliomyelitis eradication. Wkly Epidemiol Rec. 71: 189-194.
OMS (1990). Guide des méthodes de laboratoire pour le diagnostic de la poliomyélite. WHO/EPI/CDC/POLIO/90.1. 